Artigo Original

Original Article

Letícia Ribeiro Pifaia ${ }^{1}$

Glaucya Madazio²

Mara Behlau²

Descritores

Voz

Acústica da fala

Qualidade da voz

Percepção auditiva

Distúrbio da voz

Keywords

Voice

Acoustic

Voice quality

Auditory perception

Voice disorders

Endereço para correspondência:

Letícia Ribeiro Pifaia

R. Correia de Lemos, 327, Apto 81, Chácara Inglesa, São Paulo (SP), Brasil, CEP: 04140-000.

E-mail: le_pifaia@yahoo.com.br

Recebido em: 24/12/2010

Aceito em: 22/9/2011

\section{Diagrama de desvio fonatório e análise perceptivo- auditiva pré e pós-terapia vocal}

\author{
Phonatory Deviation Diagram pre and post vocal \\ rehabilitation
}

\section{RESUMO}

Objetivo: Verificar se o Diagrama de Desvio Fonatório (DDF) demonstra a mudança vocal obtida por meio da terapia fonoaudiológica e se acompanha as alterações da análise perceptivo-auditiva. Métodos: Foram analisadas amostras da vogal sustentada / $\varepsilon /$ de 34 indivíduos, com diversos tipos de disfonia, nos momentos pré e pós-terapia. As vozes foram submetidas à análise acústica pelo DDF e perceptivo-auditiva pela escala analógica visual (EAV) de 100 pontos. No DDF, as vozes foram classificadas quanto à densidade (concentrada ou ampliada), à forma (horizontal, vertical ou circular) e à localização nos quadrantes do diagrama (inferior esquerdo $=1$; inferior direito $=2$; superior direito $=3$ e superior esquerdo $=4$ ). Procedeu-se à análise estatística dos parâmetros acústicos, da distribuição das amostras e da correlação entre a análise acústica e a auditiva, nos momentos pré e pós-terapia. Resultados: Os dados acústicos mostraram melhora na situação pós-terapia. A densidade do DDF não se diferenciou nos dois momentos. Já as formas diferenciaram-se nos dois momentos $(\mathrm{p}=0,031)$, sendo que a vertical caracterizou as amostras no momento pré-terapia $(12 ; 35,29 \%)$, e a circular (7; 20,59\%) no momento pós-terapia. No momento pré-terapia, as amostras estavam distribuídas em três dos quatro quadrantes; no pós-terapia, 90\% localizaram-se no quadrante 1 (área de normalidade). A média da avaliação auditiva passou de grau moderado a leve-moderado $(\mathrm{p}<0,001)$. Houve diferença entre a localização no quadrante e a avaliação auditiva $(\mathrm{p}<0,001)$. Conclusão: $\mathrm{O}$ DDF é útil para identificar mudanças em relação à terapia vocal, correlacionando-se à avaliação perceptivo-auditiva.

\begin{abstract}
Objective: Verify whether PDD reflects vocal changes obtained by vocal rehabilitation and whether it reflects the changes in perceptual analysis. Methods: Samples of the sustained vowel / $\varepsilon /$, pre and post-therapy, from 34 individuals with different dysphonia diagnosis were collected. The voices were acoustically analyzed by the PDD and an auditory perceptual analysis was performed using a visual analog scale (VAS) of 100 points. The acoustic samples were classified according to PDD density (concentrate or spread), shape (horizontal, vertical or circular) and position within the quadrants of the graph (lower left $=1,=$ lower right $=2$, upper right $=3$ and upper left $=4$ ). Statistical analysis of acoustic parameters, distribution of samples and correlation between acoustic analysis and auditory perceptual analysis were performed. Results: Acoustic data showed improvement after therapy. Density did not differ in the two moments, while shape showed differences pre and post-therapy $(\mathrm{p}=0.031)$. The vertical form characterized pre-therapy samples $(12,35.29 \%)$, and the circular form was associated to post-therapy samples $(7,20.59 \%)$. In pre-treatment condition, samples were distributed in three of the four quadrants, while in post-therapy, $90 \%$ were located in the first quadrant (normality area). The average perceptual deviation reduced from moderate to mild-moderate $(\mathrm{p}<0.001)$. There is a relationship between quadrant location and perceptual analysis $(\mathrm{p}<0.001)$. Conclusion: PDD is useful to identify changes acquired with voice therapy and correlates with perceptual voice analysis.
\end{abstract}

Trabalho realizado no Centro de Estudos da Voz - CEV- São Paulo (SP), Brasil.

(1) Especialista em Voz pelo Conselho Federal de Fonoaudiologia.

(2) Curso de Especialização em Distúrbios da Comunicação Humana, Universidade Federal de São Paulo UNIFESP - São Paulo(SP), Brasil.

(3) Curso de Especialização em Voz, Centro de Estudos da Voz - CEV - São Paulo (SP), Brasil.

Conflito de interesse: nada a declarar. 


\section{INTRODUÇÃO}

A produção da voz envolve aspectos complexos multidimensionais, e por isso deve ser analisada em suas diferentes perspectivas $^{(1,2)}$. As análises perceptivo-auditiva e acústica são as principais ferramentas da avaliação vocal.

As análises acústica e perceptivo-auditiva são importantes instrumentos clínicos que se complementam para o monitoramento e acompanhamento do desenvolvimento dos pacientes ao longo do processo terapêutico. A análise acústica, considerada como avaliação objetiva tornou-se, recentemente, uma realidade em muitas clínicas fonoaudiológicas, depois da introdução e disponibilidade de programas de análise acústica da voz de baixo custo. Atualmente, não há motivos financeiros para a não realização da análise acústica associada à auditiva em pacientes com distúrbio vocal ${ }^{(3)}$. Quanto à análise perceptivo-auditiva, ela fornece informações importantes sobre a anatomofisiologia da laringe, presença, grau e tipo de desvio vocal, sendo considerada como subjetiva, porém soberana e padrão ouro para a avaliação vocal ${ }^{(4-6)}$.

A análise acústica permite a extração de medidas isoladas e combinadas. Contudo, medidas acústicas isoladas oferecem informações parciais e não podem ser representativas da qualidade vocal como um todo. Pesquisas recentes ${ }^{(7,8)}$ mostraram que uma associação entre medidas auditivas e acústicas, não excludentes e sim complementares, pode ser mais representativa da voz em questão e também mais útil, uma vez que se trata de importante ferramenta de monitoramento e acompanhamento de pacientes em treinamento vocal.

O Diagrama do Desvio Fonatório (DDF), do programa Voxmetria (CTS Informatica), é o recurso da análise acústica que usa parâmetros combinados em uma apresentação gráfica. O diagrama foi originalmente proposto por um grupo alemão, sob o nome de Diagrama de Rouquidão ${ }^{(7,9,10)}$. Diferentemente de outros tipos de análise acústica da voz, o DDF correlaciona quatro medidas acústicas, por meio de um gráfico bidimensional: no eixo horizontal relaciona as medidas de jitter, shimmer e suas correlações; no eixo vertical, apresenta as medidas do GNE (glottal-to-noise excitation ratio). O DDF tem sido utilizado na prática clínica para fornecer uma descrição mais confiável da qualidade vocal, capaz de acompanhar a evolução do paciente na terapia de voz e identificar a redução dos desvios vocais, além de demonstrar a distribuição da amostra vocal em direção à área de normalidade proposta pelo próprio programa ${ }^{(8)}$.

O DDF diferencia grupos disfônicos e indica diferentes mecanismos fonatórios presentes na produção vocal, facilitando ainda seu monitoramento durante o processo terapêutico ${ }^{(7-10)}$. Além disso, contribui para a identificação de diferentes tipos e graus de desvio ${ }^{(8)}$. Portanto, o DDF pode ser reputado como um recurso de avaliação acústica por oferecer dados complementares à extração de parâmetros isolados, tradicionalmente utilizados na clínica fonoaudiológica.

A apresentação visual do diagrama, associada à caracterização dos diferentes graus do desvio vocal, permite que ele seja utilizado no acompanhamento da evolução do paciente na terapia de voz. Este recurso é capaz de documentar o resultado da terapia fonoaudiológica de forma clara e simples, além de permitir uma fácil compreensão dos resultados, tanto para paciente e terapeuta, quanto para a equipe de saúde envolvida no processo.

Assim, o presente trabalho pretendeu verificar se o diagrama de desvio fonatório é capaz de apontar as mudanças de voz obtidas na terapia vocal e analisar a correlação entre a avaliação perceptivo-auditiva e a distribuição de amostras vocais no diagrama.

\section{MÉTODOS}

Esta pesquisa retrospectiva foi aprovada pelo Comitê de Ética do Centro de Estudos da Voz (CEV), sob o parecer de $n^{\circ} 1416 / 08$. Foram analisados 34 pares de registros vocais, nos momentos pré e pós-terapia, de um banco clínico de dados. As amostras foram coletadas de pacientes com queixa de voz e diagnóstico definido de disfonia, independentemente do tipo e do grau de alteração, de ambos os gêneros, sendo 13 mulheres e 21 homens, entre 27 e 85 anos (média de 49,02 anos), selecionados aleatoriamente entre os que buscaram e concluíram atendimento clínico no ano de 2009.

Todos os pacientes procuraram por atendimento no centro fonoaudiológico, e, por terem sido atendidos individualmente por duas fonoaudiólogas especialistas em voz, já possuíam exame otorrinolaringológico com hipótese diagnóstica estabelecida e encaminhamento médico para terapia vocal (disfonia funcional, alteração estrutural mínima (AEM), presbifonia, edema de Reinke, leucoplasia, granuloma, nódulos, laringite aguda, paresia, cordectomia, paralisia e pós-operatório de lesões benignas). Foram incluídas no estudo as amostras pertencentes aos pacientes que finalizaram o atendimento proposto, com registros completos referentes ao momento de avaliação (pré-terapia) e sessão de alta fonoaudiológica (pós-terapia). Não foram levadas em consideração algumas características do atendimento, como número e duração das sessões. No entanto, todos os pacientes foram submetidos a um programa de tratamento vocal individualizado, com o uso, sempre que possível, de técnicas com base científica ${ }^{(11-15)}$. A base do atendimento foi direcionada à higiene vocal, trabalho de psicodinâmica e treinamento vocal, com exercícios realizados nas sessões de atendimento e orientados para casa ${ }^{(16)}$.

O material de fala empregado nas análises foi a vogal / $/$ / sustentada, em frequência e intensidade vocais habituais, gravado em dois momentos diferentes: pré e pós-terapia vocal. O momento de pré-terapia vocal constituiu-se na sessão da avaliação vocal, quando os pacientes procuraram por atendimento, e o pós compreendeu a sessão de alta fonoaudiológica. As amostras foram gravadas e editadas no programa VoxMetria 2,7 (CTS Informática), eliminando-se o primeiro segundo da emissão, marcado por instabilidade natural, sendo analisados apenas os três segundos subsequentes.

$\mathrm{O}$ registro das vozes ocorreu em ambiente silencioso, com os participantes sentados e com o uso de microfone unidirecional de pedestal, marca Radioshack Electrec, condenser, model 33-3007, posicionado a $45^{\circ}$ da boca do paciente. As vozes foram gravadas diretamente em um computador Dell Dimension 4600 Pentium 4, com auxílio de um pré-amplificador (Tiger-DRS). 
As amostras vocais foram submetidas à avaliação acústica e perceptivo-auditiva, tendo sido a análise acústica realizada por meio do programa VoxMetria versão 2.7, da CTS Informática, no módulo Qualidade Vocal. A análise relacionou-se à extração automática dos parâmetros acústicos que compõem o DDF, a saber: jitter (perturbação da frequência fundamental, medido em porcentagem, por meio do PPQ - Period Perturbation Quotient, com valor limite de normalidade em 0,6\%); shimmer (perturbação de amplitude, em porcentagem, medido pelo EPQ - Energy Pertturbation Quotient, com valor limite de normalidade em 6,5\%); coeficiente de correlação (variação da forma da onda calculada por meio de algoritmo específico, o parâmetro WMC - Waverform Matching Coefficient, com valor limite de normalidade em 1); proporção sinal glótico/ ruído excitado - GNE (Glottal-to-Noise Excitation - cálculo do ruído em uma série de pulsos, o qual indica se o sinal vocal está sendo originado pela vibração das pregas vocais ou pela corrente de ar turbulenta gerada no trato vocal ${ }^{(9)}$, medido em $\mathrm{dB}$, com valor de normalidade maior ou igual a $0,5 \mathrm{~dB}$ ).

A análise da configuração da distribuição das amostras vocais no DDF visou avaliá-las quanto à densidade, forma e localização nos quadrantes do gráfico. A densidade foi classificada em concentra$\mathrm{da}$ - quando a distribuição dos pontos no diagrama apresentava-se no espaço de um quadrado estabelecido pelo próprio diagrama; ou ampliada — se os pontos distribuídos localizavam-se em mais de um quadrado. A configuração da forma foi classificada em horizontal, vertical ou circular, independentemente da densidade. Assim, caso a distância entre os pontos na coordenada $X$ fosse maior do que a distância entre os pontos na coordenada $\mathrm{Y}(\mathrm{X}>\mathrm{Y})$, a forma seria classificada como horizontal; se a distância entre os pontos na coordenada $\mathrm{X}(\mathrm{X}<\mathrm{Y})$ fosse menor do que na $\mathrm{Y}$, seria classificada como vertical; e, se a distância entre os pontos fosse aproximadamente igual, nas coordenadas $\mathrm{XeY},(\mathrm{X} \approx \mathrm{Y})$, seria classificada como circular (Figura1). ODDF foi dividido didaticamente em quatro quadrantes: inferior esquerdo - quadrante 1; o inferior direito - quadrante 2; superior direito - quadrante 3 ; e superior esquerdo - quadrante $4^{(8)}$ (Figura 2). O quadrante 1 compreende a área de normalidade proposta pelo próprio programa.

As vozes foram apresentadas aos pares, de forma aleatória, quanto à condição pré e pós-terapia vocal. Houve a repetição de $10 \%$ das amostras. A avaliação do grau geral de desvio da voz foi realizada por meio de uma escala analógico-visual (EAV), de 100 pontos (parâmetro "G", do CAPE-V) $)^{(17)}$, sendo que: de 0 a 35,5 pontos correspondeu à faixa de variabilidade normal da qualidade vocal (VNQV); entre 35,6 a 50,5 a de grau leve a moderado; entre 50,6 a 90,5 a de grau moderado, e com valor acima de 90,5 pontos a grau intenso. Tais valores foram baseados em estudo anterior realizado com vozes brasileiras ${ }^{(18)}$, cujos resultados foram semelhantes aos obtidos no estudo internacional que propôs a escala ${ }^{(19)}$.

A avaliação perceptivo-auditiva foi realizada por uma fonoaudióloga especialista em voz, com 14 anos de experiência na área. A confiabilidade elevada da juíza, tanto para as avaliações das amostras pré-terapia $(\mathrm{p}=0,317)$, quanto para as de pós-terapia $(\mathrm{p}=0,414)$, foi verificada por meio da aplicação do Teste dos Postos Sinalizados de Wilcoxon, com nível de significância de $5 \%(0,050)$.
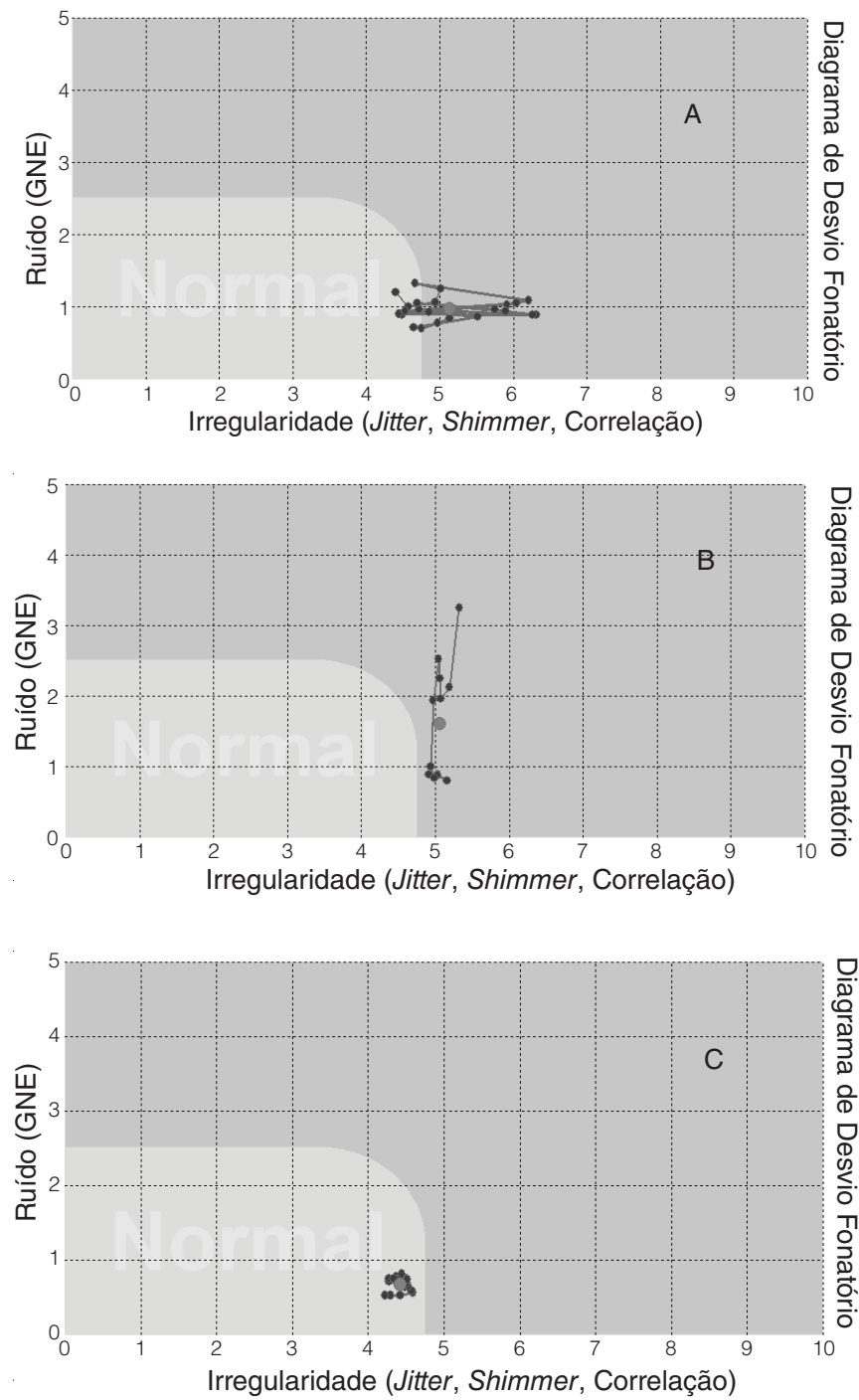

Legenda: $\mathrm{A}=$ amostra vocal de densidade ampliada e forma horizontal; $\mathrm{B}=$ amostra vocal de densidade ampliada e forma vertical; $\mathrm{C}=$ amostra vocal de densidade concentrada e forma circular

Figura 1. Exemplo de classificação da distribuição das amostras no Diagrama de Desvio Fonatório

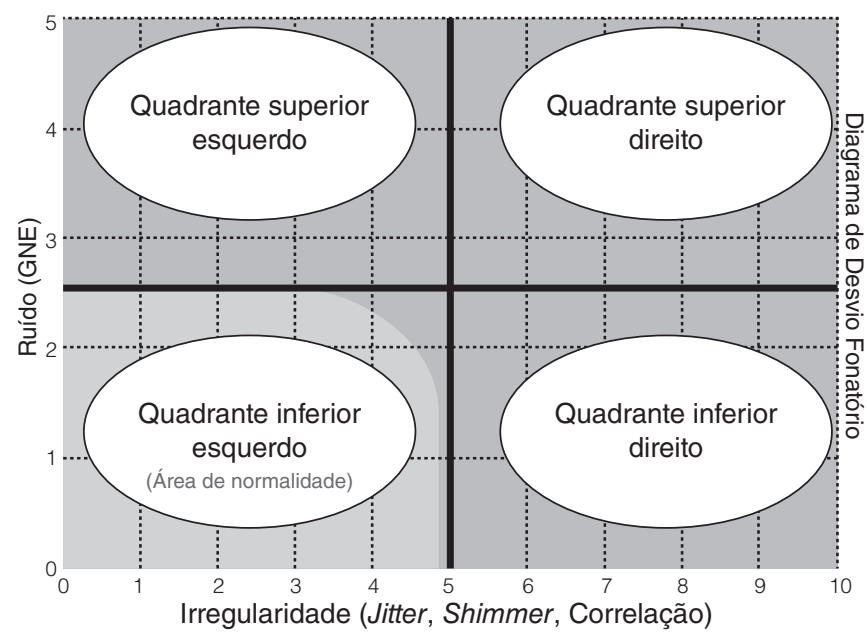

Figura 2. Divisão didática do Diagrama de Desvio Fonatório em quadrantes 
Os resultados foram submetidos a um tratamento estatístico (SPSS - Statistical Package for Social Sciences, 13.0). A aplicação do Teste dos Postos Sinalizados de Wilcoxon foi adotada para verificação de possíveis diferenças entre os dois momentos de observação citados, para as variáveis de interesse, como jitter, shimmer, GNE (denominado proporção sinal glótico/ruído excitado) e para a análise perceptivo-auditiva nos momentos pré e pós-terapia. Para a comparação entre densidade, forma e quadrantes, pré e pós-terapia, aplicou-se o Teste da Estatística V de Cramer, e para analisar a relação entre o quadrante do diagrama e o grau geral de desvio vocal, o Teste de Qui-quadrado. O nível de significância adotado foi de 5\%.

A correlação de Spearman serviu para medir a relação entre as variáveis nos dois grupos, pré e pós-terapia vocal. A Correlação de Spearman baseia-se na ordenação de duas variáveis, sem qualquer restrição quanto à distribuição de valores, ou seja, é mais utilizada para dados não paramétricos. Foi produzida uma Matriz de Correlação pela determinação dos sinais de correlação (positivo ou negativo) e sua qualidade (qualidade $<20 \%=$ péssima; 21 a $40 \%=$ ruim; 41 a 60\%=regular; 61 a $80 \%=$ boa; acima de $81 \%=$ ótima). Optou-se por analisar apenas as correlações de qualidade boa e ótima, ou seja, acima de $61 \%$. O Teste de Correlação validou os resultados, testando o coeficiente entre as variáveis.

\section{RESULTADOS}

Todos os parâmetros acústicos estudados apresentaram diferença nos momentos pré e pós-terapia. Os valores obtidos no momento pós-terapia foram mais próximos aos valores da normalidade estabelecidos pelo próprio programa (Tabela 1).

Houve diferença $(\mathrm{p}=0,031)$ na configuração da forma, entre os dois momentos estudados (Tabela 2). A forma horizontal foi a de maior ocorrência, tanto no momento pré quanto no pós-terapia vocal $(61,76$ e 58,82\%, respectivamente). No pré-terapia, a forma vertical teve a segunda maior ocorrência $(35,29 \%)$, e houve apenas uma ocorrência de forma circular. Já no momento de pós-terapia, as formas vertical e circular apresentaram a mesma ocorrência $(20,59 \%)$. Assim, no pós-terapia houve redução da ocorrência da forma vertical e aumento da forma circular (Tabela 2).

Houve diferença $(\mathrm{p}=0,019)$ na localização das amostras vocais nos momentos estudados(Tabela 3). No momento pré-terapia vocal, as amostras foram distribuídas nos quadrantes 1 e 3 (64,71 e 29,41\%, respectivamente). Já no pós-terapia vocal, mais de $90 \%$ das amostras foram localizadas no quadrante 1 (Figura 3 ).

Houve relação entre o grau do desvio vocal e a localização das amostras $(\mathrm{p}<0,001)$, em ambos os momentos estudados (Tabela 4). Todas as vozes com desvio intenso localizaram-se no quadrante 3 . As vozes avaliadas, com desvios dentro da faixa de normalidade da qualidade vocal ou com desvios de grau leve a moderado, localizaram-se no quadrante 1 . As vozes com desvio moderado apresentaram ampla distribuição, ou seja, localizaram-se nos quadrantes 1, 2 e 3 . Estas vozes, no momento pós-terapia vocal, dividiram-se entre os quadrantes 1 e 2 .

Foi realizada a matriz de correlação entre os parâmetros acústicos, a localização no DDF e a análise auditiva (Tabela 5). No

Tabela 1. Parâmetros acústicos do Diagrama de Desvio Fonatório, nos momentos pré e pós-terapia vocal

\begin{tabular}{|c|c|c|c|c|c|}
\hline Va VariVariáveis & Média & Desvio-padrão & Mínimo & Máximo & Valor de $p$ \\
\hline \multicolumn{6}{|l|}{ Jitter } \\
\hline Pré-terapia & 3,01 & 5,74 & 0,07 & 17,30 & \multirow{2}{*}{$<0,001^{\star}$} \\
\hline Pós-terapia & 0,27 & 0,76 & 0,05 & 4,52 & \\
\hline \multicolumn{6}{|l|}{ Shimmer } \\
\hline Pré-terapia & 10,92 & 11,52 & 1,52 & 43,64 & \multirow{2}{*}{$<0,001^{\star}$} \\
\hline Pós-terapia & 4,49 & 3,51 & 1,44 & 17,31 & \\
\hline \multicolumn{6}{|l|}{ Correlação } \\
\hline Pré-terapia & 0,89 & 0,18 & 0,41 & 1,00 & \multirow{2}{*}{$<0,001^{*}$} \\
\hline Pós-terapia & 0,98 & 0,03 & 0,85 & 1,00 & \\
\hline \multicolumn{6}{|l|}{ Proporção GNE } \\
\hline Pré-terapia & 0,64 & 0,29 & 0,07 & 0,94 & \multirow{2}{*}{$0,002^{*}$} \\
\hline Pós-terapia & 0,82 & 0,13 & 0,48 & 0,97 & \\
\hline
\end{tabular}

*Valores significativos $(p<0,050)-$ Teste de Wilcoxon

Tabela 2. Forma no Diagrama de Desvio Fonatório, nos momentos pré e pós-terapia vocal

\begin{tabular}{|c|c|c|c|c|c|c|c|c|c|}
\hline \multirow{3}{*}{ Forma pré } & \multicolumn{8}{|c|}{ Forma pós } & \multirow{3}{*}{ Valor de $p$} \\
\hline & \multicolumn{2}{|c|}{ Circular } & \multicolumn{2}{|c|}{ Horizontal } & \multicolumn{2}{|c|}{ Vertical } & \multicolumn{2}{|c|}{ Total } & \\
\hline & $n$ & $\%$ & $\mathrm{n}$ & $\%$ & $n$ & $\%$ & $\mathrm{n}$ & $\%$ & \\
\hline Circular & 1 & 2,94 & 0 & 0 & 0 & 0 & 1 & 2,94 & \\
\hline Horizontal & 3 & 8,82 & 16 & 47,06 & 2 & 5,88 & 21 & 61,76 & $0,031^{*}$ \\
\hline Vertical & 3 & 8,82 & 4 & 11,76 & 5 & 14,71 & 12 & 35,29 & \\
\hline Total & 7 & 20,59 & 20 & 58,82 & 7 & 20,59 & 34 & 100 & \\
\hline
\end{tabular}

* Valores significativos $(\mathrm{p}<0,050)-$ Teste $V$ de Cramer 


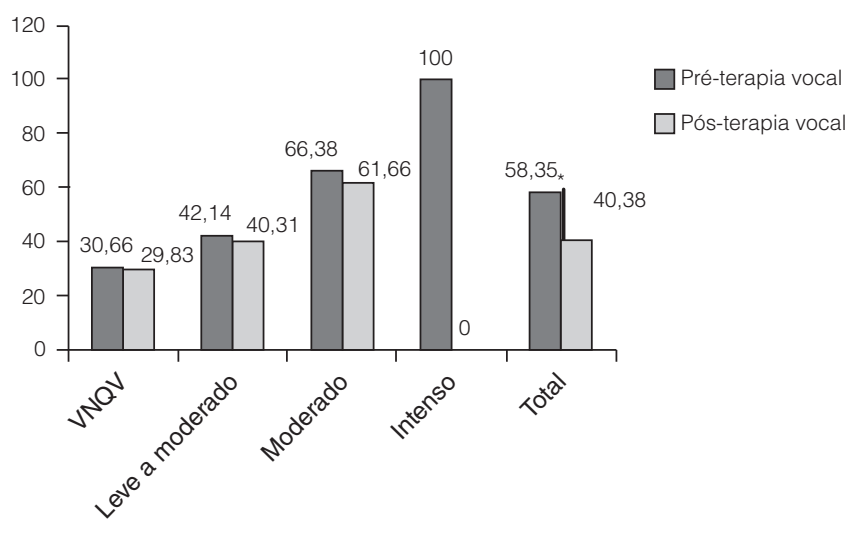

*Valores significativos $(p<0,001)$ - Teste de Wilcoxon

Legenda: VNQV = variabilidade normal da qualidade vocal

Figura 3. Média do grau de desvio da qualidade vocal à avaliação perceptivo-auditiva na EAV, nos momentos pré e pós-terapia vocal

momento pré-terapia, o valor de correlação apresentou-se entre 61 a $80 \%$, sendo considerado bom. Já no momento pós-terapia, posicionou-se entre 21 e $40 \%$, sendo considerado ruim.

Está ilustrado a média do grau de desvio da alteração vocal, obtida por meio da escala analógico-visual, nos momentos pré e pós-terapia vocal, na Figura 3. No pré-terapia, a média do grau de desvio vocal do grupo estudado foi de 58,35 pontos na EAV, valor este tido como grau moderado; no pós-terapia vocal, a média do grau de desvio reduziu-se a 40,38 pontos na
EAV, sendo considerada como de grau leve a moderado. Houve diferença entre os momentos pré e pós-terapia vocal $(\mathrm{p}<0,001)$.

\section{DISCUSSÃO}

O diagrama de desvio fonatório é um instrumento recente, ainda pouco explorado, porém promissor. Oferece uma análise acústica diferente das anteriores, permitindo a extração dos parâmetros acústicos com outra opção algorítmica ${ }^{(10,20,21)}$, além da análise combinada deste parâmetros.

Os programas mais empregados na avaliação acústica vocal apresentam grandes limitações na análise de vozes com maiores graus de desvio, pela própria opção algorítmica utilizada na extração dos valores de perturbação ${ }^{(10,21)}$. Os algoritmos, relacionados com as medidas de periodicidade da onda sonora, utilizados no diagrama de desvio fonatório, foram propostos pelos criadores do diagrama ${ }^{(7,9,10)}$ para contemplar os sinais vocais com maiores desvios, bastante frequentes na clínica fonoaudiológica.

Mais importante do que realizar múltiplas avaliações em pacientes com queixa vocal é compreender como os resultados se relacionam. A correlação entre o comportamento vocal e as análises perceptivo-auditiva e acústica da voz facilita a compreensão do mecanismo anatomofisiológico do sistema fonatório apresentado pelo paciente e, consequentemente, viabiliza a elaboração de um plano de terapia mais eficiente e direcionado. O DDF facilita essa análise combinada e sugere prováveis

Tabela 3. Localização das amostras nos quadrantes do Diagrama de Desvio Fonatório, nos momentos pré e pós-terapia vocal

\begin{tabular}{|c|c|c|c|c|c|c|c|c|c|c|c|}
\hline \multirow{3}{*}{ Quadrante pré } & \multicolumn{10}{|c|}{ Quadrante pós } & \multirow{3}{*}{ Valor de $p$} \\
\hline & \multicolumn{2}{|c|}{1} & \multicolumn{2}{|c|}{2} & \multicolumn{2}{|c|}{3} & \multicolumn{2}{|c|}{4} & \multicolumn{2}{|c|}{ Total } & \\
\hline & $\mathrm{n}$ & $\%$ & $\mathrm{n}$ & $\%$ & $\mathrm{n}$ & $\%$ & $\mathrm{n}$ & $\%$ & $\mathrm{n}$ & $\%$ & \\
\hline 1 & 22 & 64,71 & 0 & 0 & 0 & 0 & 0 & 0 & 22 & 64,71 & \multirow{4}{*}{$0,019^{\star}$} \\
\hline 2 & 2 & 5,88 & 0 & 0 & 0 & 0 & 0 & 0 & 2 & 5,88 & \\
\hline 3 & 7 & 20,59 & 3 & 8,82 & 0 & 0 & 0 & 0 & 10 & 29,41 & \\
\hline 4 & 0 & 0 & 0 & 0 & 0 & 0 & 0 & 0 & 0 & 0 & \\
\hline Total & 31 & 91,18 & 3 & 8,82 & 0 & 0 & 0 & 0 & 34 & 100 & \\
\hline
\end{tabular}

* Valores significativos $(p<0,050)-$ Teste de $\chi^{2}$

Tabela 4. Relação entre quadrantes no Diagrama de Desvio Fonatório e os graus de desvio vocal, nos momentos pré e pós-terapia

\begin{tabular}{|c|c|c|c|c|c|c|c|c|c|c|c|}
\hline \multirow{2}{*}{ Quadrante } & \multicolumn{2}{|c|}{ VNQV } & \multicolumn{2}{|c|}{ Leve a moderado } & \multicolumn{2}{|c|}{ Moderado } & \multicolumn{2}{|c|}{ Intenso } & \multicolumn{2}{|c|}{ Total } & \multirow{2}{*}{ Valor de $p$} \\
\hline & $\mathrm{n}$ & $\%$ & $n$ & $\%$ & $\mathrm{n}$ & $\%$ & $\mathrm{n}$ & $\%$ & $\mathrm{n}$ & $\%$ & \\
\hline \multicolumn{12}{|l|}{ Pré } \\
\hline 1 & 3 & 8,82 & 15 & 44,12 & 4 & 11,76 & 0 & 0 & 22 & 64,7 & \multirow{4}{*}{$<0,001^{*}$} \\
\hline 2 & 0 & 0 & 0 & 0 & 2 & 5,88 & 0 & 0 & 2 & 5,88 & \\
\hline 3 & 0 & 0 & 0 & 0 & 5 & 14,71 & 5 & 14,71 & 10 & 29,42 & \\
\hline 4 & 0 & 0 & 0 & 0 & 0 & 0 & 0 & 0 & 0 & 0 & \\
\hline Total & 3 & 8,82 & 15 & 44,12 & 11 & 32,35 & 5 & 14,71 & 34 & 100 & \\
\hline \multicolumn{12}{|l|}{ Pós } \\
\hline 1 & 12 & 35,29 & 16 & 47,06 & 3 & 8,82 & 0 & 0 & 31 & 91,17 & \multirow{4}{*}{$<0,001^{*}$} \\
\hline 2 & 0 & 0 & 0 & 0 & 3 & 8,82 & 0 & 0 & 3 & 8,82 & \\
\hline 3 & 0 & 0 & 0 & 0 & 0 & 0 & 0 & 0 & 0 & 0 & \\
\hline 4 & 0 & 0 & 0 & 0 & 0 & 0 & 0 & 0 & 0 & 0 & \\
\hline Total & 12 & 35,29 & 16 & 47,06 & 6 & 17,64 & 0 & 0 & 34 & 100 & \\
\hline
\end{tabular}

* Valores significativos $(\mathrm{p}<0,050)-$ Teste de Wilcoxon

Legenda: VNQV = variabilidade normal da qualidade vocal 
Tabela 5. Matriz de correlação entre os parâmetros acústicos, a localização no Diagrama de Desvio Fonatório e análise perceptivo-auditiva

\begin{tabular}{|c|c|c|c|c|c|c|c|c|}
\hline Pré-terapia & & Jitter & Shimmer & Correlação & Irregularidade & GNE & Quadrante & EAV \\
\hline \multirow{2}{*}{ Shimmer } & Corr & $63,5 \%$ & & & & & & \\
\hline & Valor de $p$ & $<0,001$ & & & & & & \\
\hline \multirow{2}{*}{ Correlação } & Corr & $-88,5 \%$ & $-73,7 \%$ & & & & & \\
\hline & Valor de $p$ & $<0,001$ & $<0,001$ & & & & & \\
\hline \multirow{2}{*}{ Irregularidade } & Corr & $85,7 \%$ & $86 \%$ & $-95,3 \%$ & & & & \\
\hline & Valor de $p$ & $<0,001$ & $<0,001$ & $<0,001$ & & & & \\
\hline \multirow{2}{*}{ GNE } & Corr & $-75,8 \%$ & $-54,8 \%$ & $75,9 \%$ & $-69 \%$ & & & \\
\hline & Valor de $p$ & $<0,001$ & 0,001 & $<0,001$ & $<0,001$ & & & \\
\hline \multirow{2}{*}{ Quadrante } & Corr & $79 \%$ & $77,5 \%$ & $-82 \%$ & $80,9 \%$ & $78,2 \%$ & & \\
\hline & Valor de $p$ & $<0,001$ & $<0,001$ & $<0,001$ & $<0,001$ & $<0,001$ & & \\
\hline \multirow{2}{*}{ EAV } & Corr & $68 \%$ & $60,9 \%$ & $-79,7 \%$ & $75,1 \%$ & $-78,7 \%$ & $77,4 \%$ & \\
\hline & Valor de $p$ & $<0,001$ & $<0,001$ & $<0,001$ & $<0,001$ & $<0,001$ & $<0,001$ & \\
\hline \multirow{2}{*}{ Grau de desvio } & Corr & $68,4 \%$ & $63,8 \%$ & $80 \%$ & $76,7 \%$ & $-76,4 \%$ & $76,4 \%$ & $92,1 \%$ \\
\hline & Valor de $p$ & $<0,001$ & $<0,001$ & $<0,001$ & $<0,001$ & $<0,001$ & $<0,001$ & $<0,001$ \\
\hline \multicolumn{9}{|l|}{ Pós-terapia } \\
\hline \multirow{2}{*}{ Shimmer } & Corr & $30,6 \%$ & & & & & & \\
\hline & Valor de $p$ & 0,078 & & & & & & \\
\hline \multirow{2}{*}{ Correlação } & Corr & $-60,9 \%$ & $-63,2 \%$ & & & & & \\
\hline & Valor de $p$ & $<0,001$ & $<0,001$ & & & & & \\
\hline \multirow{2}{*}{ Irregularidade } & Corr & $66,5 \%$ & $75,9 \%$ & $-92,4 \%$ & & & & \\
\hline & Valor de $p$ & $<0,001$ & $<0,001$ & $<0,001$ & & & & \\
\hline \multirow{2}{*}{ GNE } & Corr & $-19,3 \%$ & $-36,5 \%$ & $37,5 \%$ & $-39 \%$ & & & \\
\hline & Valor de $p$ & 0,274 & 0,034 & 0,029 & 0,23 & & & \\
\hline \multirow{2}{*}{ Quadrante } & Corr & $48,3 \%$ & $49,2 \%$ & $-51,8 \%$ & $49,2 \%$ & $-40,8 \%$ & & \\
\hline & Valor de $p$ & 0,004 & 0,003 & 0,002 & 0,003 & 0,017 & & \\
\hline \multirow{2}{*}{ EAV } & Corr & $34,6 \%$ & $28,0 \%$ & $-33,1 \%$ & $37,3 \%$ & $-35,7 \%$ & $49,2 \%$ & \\
\hline & Valor de $p$ & 0,045 & 0,108 & 0,056 & 0,030 & 0,038 & 0,003 & \\
\hline \multirow{2}{*}{ Grau de desvio } & Corr & $40,9 \%$ & $28,6 \%$ & $-28,3 \%$ & $35,9 \%$ & $-29,7 \%$ & $48,2 \%$ & $92,2 \%$ \\
\hline & Valor de $p$ & 0,016 & 0,102 & 0,105 & 0,037 & 0,088 & 0,004 & $<0,001$ \\
\hline
\end{tabular}

Teste Correlação de Spearman

Legenda: GNE = glottal-to-noise excitation ratio; $\mathrm{EAV}$ = escala analógico-visual

mecanismos fonatórios empregados pelo paciente durante a produção da voz.

Na literatura, alguns estudos relacionam os dados acústicos a certos aspectos da avaliação perceptivo-auditiva, mas nem sempre é encontrada correlação direta entre essas análises ${ }^{(7,9,10)}$. É possível que uma dimensão específica da qualidade vocal tenha uma ou mais pistas acústicas para sua percepção, do momento em que a avaliação auditiva é mais abrangente do que a análise acústica ${ }^{(22)}$. Uma série de experimentos tentou determinar quais pistas estariam envolvidas nas dimensões específicas da qualidade vocal. Algumas características acústicas do sinal vocal são achados comuns de certas publicações, como as medidas de perturbação da frequência fundamental ou o ruído, embora estes achados estejam relacionados aos modelos de produção de fala e não de sua percepção ${ }^{(5)}$. Assim, a busca por pistas acústicas da qualidade vocal deve ir além das medidas, já propostas, para quantificar os aspectos da produção vocal ${ }^{(5,7,20,21,23-25)}$.

Observou-se que os dados acústicos fornecidos pelo relatório estatístico do programa VoxMetria foram diferentes nos momentos pré e pós-terapia vocal (Tabela 1). Os valores das médias dos parâmetros acústicos estavam na faixa esperada. A grande parte das amostras vocais apresentava algum grau de desvio no momento pré-terapia. Já no momento pós, as amostras foram classificadas como normal ou quase normal. Estes dados não estabelecem correlação direta entre a disfonia e um ou outro parâmetro acústico isolado ${ }^{(2,18,26)}$. Os valores dos parâmetros acústicos acompanharam a melhora da qualidade vocal, avaliada por meio da análise perceptivo-auditiva da voz.

Em relação à distribuição das amostras vocais no DDF, as configurações foram avaliadas quanto à densidade, forma e localização nos quadrantes. Quanto à densidade, não houve diferença entre os momentos pré e pós-terapia vocal. Contudo, sem diferença, e, de forma descritiva, observou-se que houve um aumento da densidade concentrada no momento de pós-terapia. Os dados do presente trabalho parecem corroborar estudo anterior ${ }^{(8)}$, que encontrou maior concentração de pontos em vozes menos alteradas. Tal fato pode ser justificado pelo tamanho reduzido da amostra, pela variedade dos casos selecionados, ou ainda pelo grau da disfonia, uma vez que mais da metade das vozes, mesmo no momento pré-terapia vocal, apresentava variabilidade normal da qualidade vocal ou desvio de leve a moderado.

A mudança na configuração da forma das distribuições das amostras vocais durante o processo terapêutico pôde ser observada. A forma horizontal foi a de maior ocorrência, 
independentemente do momento estudado. A forma circular foi característica de vozes no momento pós-terapia vocal, uma vez que tal forma foi observada em apenas uma amostra no momento pré-terapia. Quando se atinge um equilíbrio entre a rugosidade e a soprosidade durante a fonação, espera-se esta forma. Já a forma vertical, menos frequente em vozes adaptadas ${ }^{(25)}$, ocorreu em menor número no momento pós-terapia. Cumpre lembrar que, mesmo as amostras vocais, avaliadas no momento pós-fonoterapia, não necessariamente apresentavam qualidade vocal dentro da faixa de variabilidade normal. Se isso tivesse ocorrido, talvez a distribuição vertical fosse ainda menor, já que tal forma é característica de vozes disfônicas.

Quanto à localização das amostras vocais nos quadrantes (Figura 3), no momento de pré-terapia vocal, observou-se que embora a maior parte delas estivesse no quadrante 1 (inferior esquerdo), algumas se dispuseram nos quadrantes 2 (inferior direito) e 3 (superior direito) (Tabela 3). Nenhuma amostra esteve localizada no quadrante 4 (superior esquerdo). Estes dados corroboram a literatura que indica que conforme o grau da disfonia diminui, as vozes aproximam-se do quadrante 1 , onde está localizada a área de normalidade proposta pelo programa $^{(8)}$. No momento pós-terapia vocal, mais de $90 \%$ das amostras estudadas localizaram-se no quadrante 1 . O restante concentrou-se no quadrante 2 , e, como já foi mencionado, vale lembrar que nem todas os pacientes apresentavam vozes adaptadas no momento da alta fonoaudiológica, o que pode apenas indicar o limite terapêutico. Das amostras localizadas no quadrante 3 , no pré-terapia vocal, a grande parte migrou para o quadrante $1 \mathrm{e}$, uma pequena parte, para o quadrante 2 . As duas vozes que se localizaram no quadrante 2 no pré-terapia migraram para o quadrante 1 . O deslocamento das amostras vocais pelos quadrantes demonstra a eficiência da terapia, com diminuição dos valores de jitter, shimmer e correlações, assim como do ruído, permitindo um ajuste fonatório mais adequado ao processo de fonação.

Além da análise acústica, as vozes também foram submetidas à avaliação perceptivo-auditiva, por meio da escala analógico-visual ${ }^{(18)}$. A média obtida para o grau de desvio vocal foi menor no momento pós-terapia vocal (de leve a moderada), e portanto, mais próxima da variabilidade normal (Figura 3). A média do grau do desvio no momento pré-terapia correspondeu ao grau moderado de desvio.

Foi avaliada a relação entre a localização das amostras vocais nos quadrantes do DDF e o grau de desvio vocal (Tabela 4). Nos momentos pré e pós-terapia vocal, de acordo com a correspondência do grau de desvio da escala analógico visual ${ }^{(18)}$, as vozes avaliadas com desvio dentro da variabilidade normal (VNQV), ou ainda com desvio em grau leve a moderado, localizaram-se no quadrante 1 , área da normalidade ${ }^{(8)}$. Houve um grande aumento das vozes avaliadas dentro da VNQV no decorrer do processo terapêutico. As vozes avaliadas com desvio em grau leve a moderado constituíram a maioria do grupo, tanto no pré quanto no pós-terapia. No pós-terapia houve um deslocamento da classificação das vozes, antes classificadas como moderadas e intensas, e agora para grau leve, em virtude de melhoria auditiva.
No momento pré-terapia, as vozes moderadas distribuíram-se nos quadrantes 1,2 e 3 , e este fato merece algumas considerações. Em primeiro lugar, vale ressaltar que a localização também é influenciada pelo tipo de $\mathrm{voz}^{(8)}$, o que não foi considerado neste estudo. Além disso, na $\mathrm{EAV}^{(18)}$, a faixa de distribuição do grau moderado de desvio é a mais extensa; vozes mais próximas do limite inferior, 50,5 pontos na EAV, localizaram-se nos quadrante 1 e 2; aquelas mais próximas do limite superior, 90,5 pontos, localizaram-se no quadrante 3 . Houve redução importante do grupo que representava vozes com desvio moderado no momento pós-terapia, e estas amostras dividiram-se entre os quadrantes 1 e 2 . Nenhuma voz foi avaliada com grau intenso de desvio no pós-terapia vocal. A avaliação perceptivo-auditiva e o deslocamento das vozes nos quadrantes reforçaram a eficiência da terapia vocal.

Também foi considerada a matriz de correlação entre os parâmetros acústicos, a localização no DDF e a análise auditiva (Tabela 5). Como esperado, houve correlação positiva entre a análise perceptivo-auditiva das vozes e os parâmetros acústicos extraídos dos mesmos sinais de áudio, tanto no pré quanto no pós-terapia, que identificaram aspectos particulares da voz avaliada. Os valores desta correlação foram maiores no pré-terapia, provavelmente favorecidos por um maior desvio vocal em tal situação.

As avaliações acústica e perceptivo-auditiva foram ferramentas importantes para avaliação e acompanhamento do paciente durante o processo terapêutico na prática clínica, principalmente como monitoramento visual para o próprio paciente ${ }^{(7,9,10)}$. Desta forma, os parâmetros analisados indicaram o resultado positivo da terapia vocal, demonstrando que o paciente foi capaz de reorganizar os ajustes motores durante a fonação, produzindo a voz de modo mais equilibrado com a terapia vocal ${ }^{(3,18,27,28)}$.

A literatura questiona a análise acústica na extração de parâmetros de vozes com elevado grau de desvio; sugere-se que, nestes casos, que seja feita a análise espectrográfica qualitativa descritiva $^{(29,30)}$. Entretanto, a opção algorítmica do DDF permite que vozes com elevados graus de desvio sejam analisadas com confiabilidade na extração das medidas ${ }^{(7,10,30)}$.

Mais do que identificar a qualidade vocal é importante quantificar a magnitude da qualidade ${ }^{(5)}$. Para o acompanhamento da terapia, ou mesmo para quantificar uma melhora no desempenho vocal, podem ser utilizadas como ferramentas complementares a extração de parâmetros acústicos, a configuração no DDF e a análise perceptivo-auditiva. Durante o monitoramento da qualidade vocal, o DDF não induz a contradições na interpretação dos resultados obtidos, mostrando-se um instrumento útil e confiável para a prática clínica, principalmente para o monitoramento do próprio paciente, que consegue visualizar a evolução da terapia por meio do deslocamento da voz no diagrama.

\section{CONCLUSÃO}

O DDF é útil para identificar mudanças durante a terapia vocal e se correlaciona com os dados da avaliação perceptivo-auditiva. 
* MB foi orientadora e responsável pelo delineamento da pesquisa organização do banco de dados e correção do texto. GM foi co-orientadora e responsável pela execução da pesquisa e correção do texto. LRP foi responsável pela pesquisa, análise dos dados e elaboração do texto.

\section{REFERÊNCIAS}

1. Takahashi H, Koike Y. Some perceptual dimensions and acoustical correlates of pathologic voices. Acta Otolaryngol. 1976;338:2-22.

2. Carding PN, Steen IN, Webb A, Mackenzie K, Deary IJ, Wilson JA. The reliability and sensitivity to change of acoustic measures of voice quality. Clin Otolaryngol. 2004;29(5):538-44.

3. Oates,J. Auditory-perceptual evaluation of disordered voice quality: pros, cons and future directions. Folia Phoniatr Logop. 2009;61(1):49-56.

4. Behlau M, Madazio G, Feijó D, Pontes P. Avaliação de voz. In: Behlau M, Voz: o livro do especialista. v.1. Rio de Janeiro: Revinter; 2001. p. 85-246.

5. Speyer R, Wieneke GH, Dejonckere PH. Documentation of progress in voice therapy: perceptual, acoustic, and laryngostroboscopic findings pretherapy and posttherapy. J Voice. 2004;18(3):325-40.

6. Patel S, Shrivastav R. Perception of dysphonic vocal quality: some thoughts and research update. Perspectives on Voice and Voice Disorders. 2007;17(2):3-6.

7. Fröhlich M, Michaelis D, Strube, HW, Kruse E. Acoustic voice analysis by means of the hoarseness diagram. J Speech Lang Hear Res. 2000;43(3):706-20.

8. Madazio G, Leão S, Behlau M. The phonatory deviation diagram: a novel objective measurement of vocal function. Folia Phoniatr Logop. 2011;63(6):305-311.

9. Michaelis D, Gramss T, Strube HW. Glottal-to-noise excitation ratio a new measure for describing pathological voices. Acta Acustica. 1997;83:700-6.

10. Fröhlich M, Michaelis D, Strube HW, Kruse E. Acoustic voice quality description: Case studies for different regions of the hoarseness diagram. In: Wittenberg T, Mergell P, Tigges M, Eysholdt U. (Eds). Advances in quantative laryngoscopy, 2 nd "Round Table". Erlangen; 1997. p. 143-150.

11. Werth K, Voigt D, Döllinger M, Eysholdt U, Lohscheller J. Clinical value of acoustic voice measures: a retrospective study. Eur Arch Otorhinolaryngol. 2010;267(8):1261-71.

12. Roy N, Weinrich B, Gray SD, Tanner K, Stemple JC, Sapienza CM. Three treatments for teachers with voice disorders: a randomized clinical trial. J Speech Lang Hear Res. 2003;46(3):670-88.

13. Lessac A. The use and training of the human voice: a Bio-Dynamic approach to vocal life. Mountain View, Mayfield; 1997.

14. Roy N, Leeper HA. Effects of the manual laryngeal musculoskeletal tension reduction technique as a treatment for functional voice disorders: perceptual and acoustic measures. J Voice. 1993;7(3):242-9.

15. Roy N, Weinrich B, Gray SD, Tanner K, Stemple JC, Sapienza CM. Three treatments for teachers with voice disorders: a randomized clinical trial. J. Speech Lang Hear Res. 2003;46(3):670-88.
16. Behlau M, Madazio G, Feijó D, Azevedo R, Gielow I, Rehder MI. Aperfeiçoamento vocal e Tratamento fonoaudiológico das disfonias. In: Behlau M, organizador. Voz: o livro do especialista. v.2. Rio de Janeiro: Revinter; 2005. p. 409-528.

17. Behlau M. Consensus auditory - perceptual evaluation of voice (CAPE-V), ASHA 2003. Rev Soc Bras Fonoaudiol. 2004;9(3):187-9.

18. Yamasaki R, Leão S, Madazio G, Padovani M, Azevedo R, Behlau M. Correspondência entre escala analógico-visual e escala numérica na avaliação perceptivo-auditiva de vozes. Rev Soc Bras Fonoaudiol. 2008;13(Suppl):1080.

19. Simberg S, Laine A, Sala E, Rönnemaa AM. Prevalence of voice disorders among future teachers. J Voice. 2000;14(2):231-5.

20. Eskenazi L, Childers DG, Hicks DM. Acoustic correlates of vocal quality. J Speech Lang Hear Res. 1990;33(2):298-306.

21. Madazio G, Leão S, Behlau M. Hoarseness diagram in voice predominantly rough, breath and strain. In: The Voice Foundation; 2006; Philadelphia. Abstract.

22. Titze I, Liang, H. Comparison of F0 extraction methods for high precision voice perturbation measurement. J Speech Hear Res 1993;36(6):1120-33

23. Kreiman J, Gerrat BR, Kempster GB, Erman A, Berke GS. Perceptual evaluation of voice quality: review, tutorial and a framework for future research. J Speech Lang Hear Res 1993;36(1):21-40.

24. Dejonckere PH. Principal components in voice pathology. J Voice. 1995;4:96-105.

25. Dejonckere PH, Lebacq J. Acoustic, perceptual, aerodynamic and anatomical correlations in voice pathology. ORL J Otorhinolaryngol Relat Spec. 1996;58(6):326-32.

26. Kreiman J, Gerrat B, Ito M. When and why listeners disagree in voice quality assessment tasks. J Acoust Soc Am. 2007;122(4):2354-64.

27. Frölich M, Michaelis D, Strube HW. Acoustic breathiness measures in the description of pathological voices. In: Proceedings ICASSP 1998, Seattle, WA; 1998. p. 937-40.

28. Rabinov CR, Kreiman J, Gerrat BR, Bielamowicz S. Comparing reliability of perceptual ratings of roughness and acoustic measures of jitter. J Speech Lang Hear Res. 1995;38(1):26-32.

29. Bielamowicz S, Kreiman J, Gerratt BR, Dauer MS, Berke, GS. Comparison of voice analysis systems for perturbation measurement. J Speech Lang Hear Res. 1996;39(1):126-34.

30. Schneider B, Denk DM, Bigenzahn W. Acoustic assessment of the voice quality before and after medialization thyroplasty using the titanium vocal fold medialization implant (TVFMI). Otolaryngol Head Neck Surg. 2003;128(6):815-22. 